\title{
Chapter 10: Ontological Relations
}

\section{Ulf Schwarz and Barry Smith}

\section{Formal Ontological Relations: What are They? What are They For?}

During our discussion of the ontological sextet and the associated classification of reality in Chapter 8, we saw that the classical Aristotelian square can be extended to encompass both particulars and universals, and both continuants and occurrents, to yield a total of six categories. We had postulated very specific relations among the entities in these categories, such as the relations of inherence, participation, instantiation, exemplification, and characterization. In this chapter, we will discuss how to characterize the relations among the entities in these categories more precisely and to define the relational expressions used in ontologies in a more rigorous and unambiguous way.

The relations mentioned are genuine formal-ontological relations. That means, first, that they are ontological, not merely logical. Certainly, as thinkers such as Frege and Boole have argued, studying logic can help us to gain some understanding of general aspects of reality (see Meixner, 1992). However, to gain a more complete understanding of this reality it is ontological relations, not logical ones, which we must study. Second, it means that the given relations are formal. They are not additional components of reality; rather, they are that which binds existing entities into larger unities (Ceusters, et al., 2006). An example of a formalontological relation in this sense is the part of relation, in which your arm, leg, head, and so forth, stand to the whole that is your body. In order to explore and understand the ontological structure of reality, it is necessary to create an assay of such important relations.

The most appropriate method used for attaining this goal is to concentrate on an ontology describing some specific domain, such as biological reality (for the moment, we will leave top-level ontologies aside). Through examples derived from a delineated area of knowledge or science, we can show which relations obtain among the entities of that domain, and how the relational expressions used in corresponding ontologies can be defined in a way that is rigorous, unambiguous, and consistent. For this purpose, we must explicitly acknowledge the existence of certain other kinds of entities, such as points in time and spatial regions, which are important for expressing simple relations in biology and thus also in ontologies of the biological domain. These entities do not appear in 
the top-level categories delineated by the ontological sextet. They appear at lower, more specified, levels of categorization.

The relations that appear at the top-level categories of the ontological sextet can be distinguished from one another on the basis of whether their relata are universals or particulars. We can distinguish three typical cases:

$<$ universal, universal $>$ : Both relata are universals. An example of this type of relation is characterization, or the subsumption (is_a) relation which obtains between the universal human and the universal mammal, such that: human is_a mammal.

<instance, universal $>$ : The first relatum is a particular, the second is a universal. An example of a relation of this type is the instantiation relation, which obtains between this particular person named Peter and the universal human, or between Peter's life and the universal life. Another example is the relation of being allergic to that exists between Peter and the universal aspirin.

$<$ instance, instance $>$ : Both relata are particulars. Examples include the inherence relation, or the participation relation which obtains between Peter's life and Peter, or also - independently of the ontological sextet the part-whole relation on the level of instances, which obtains between this particular nose (Peter's nose) and this particular head (Peter's head), and between both of these and Peter.

For what follows, we want to introduce two terminological conventions. First, in order to avoid any ambiguities, we will use italics when referring to relations which obtain only between universals, and bold face to express any relations which have among their relata at least one particular. Second, we will use expressions common in applied ontology: for example, is_a for the subsumption relation and instance_of for the instantiation relation. Third, we will confine ourselves to binary relations, though the ideas here expressed can be generalized in the obvious way to relations with any number of relata. Some relations, such as the participation relation and the part-whole relation, obtain in different forms both at the level of instances and at the level of universals: thus, we can speak of participates_in and part_of, as well as of participates_in and part_of. Where needed, we shall refer to these as instance-level and type-level relations. 
The relations in the ontological sextet also play an important role in an ontology of the biological domain. We will use both instance-level relations, and relations obtaining between instances and universals, in order to define expressions for those relations at the level of universals ${ }^{48}$ that are of special interest to ontologies in the biological domain such as the Gene Ontology. It is these type-level relations that are at the center of interest for anyone wishing to use information systems to represent the knowledge that is captured in biomedical science. Such knowledge is knowledge of universals. It is not Fury or Black Beauty that are described in zoology textbooks, but rather the type horse: not particulars, but universals and their relations of universals one to another.

Biological ontologies were developed in order to serve as controlled vocabularies for the expression of the results of biological research. Such vocabularies contain sentences of the form $A$ relation $B$, in which ' $A$ ' and ' $B$ ' are terms in a biological ontology and 'relation' is a placeholder for 'part_of' or a similar expression. Such sentences convey general information about the corresponding biological universals. The question of which entities ${ }^{49}$ count as biological universals (and which do not) cannot be answered easily. For the moment, we can content ourselves with the many examples of terms - such as cell or reproduction or axon or glycolosis - in ontologies which are used in biological literature to refer to general types of objects or processes. While unavoidable in biological research, statements about particular instances of these types (for example, a statement about the specific weight of this particular organism in a particular petri dish at some particular time) do not belong to the general claims of biological science. Accordingly, relations in biological ontologies link universals with other universals.

Instances are important to ontologies, nonetheless: for we cannot define what it is for universals to stand in a specific relation (for example, the type-level relation: part_of) without reverting to consideration of their corresponding instances. We can specify what it means for retina to be part_of eye only when we recognize that this is a statement to the effect

${ }^{48}$ To avoid unnecessarily complicated linguistic constructions, we will use 'definition of a relation' and 'definition of a relational expression' synonomously, except in places where this might result in confusion.

${ }^{49}$ In the following, the word 'entity' will be used as ontological term of art to refer to everything which, in some way, exists (all continuants, processes, functions, structures, places, times, etc., at the level of instances as well as the level of universals). 
that all instances of the universal retina stand in a part_of relation to certain corresponding instances of the universal eye. This dependence of type-level relations on relations between their corresponding instances forms the basis of our definitions below of the relational expressions that link general terms to each other. For general terms are no more than the names of universals, and science is concerned with general statements about such universals, rather than with the way the world happens to be at some time or place.

In the following, we lay out rigorous definitions of the subtype-, parthood-, participation-, and location-relation between universals. Then, we introduce a method for defining other ontological relations between entities in other domains of reality.

\section{Benefits and Problems in Defining Formal Ontological Relations}

The consistent use of rigorous definitions to characterize formal relations will be a major step toward enabling information scientists to achieve interoperability among ontologies in support of automated reasoning across data derived from multiple domains. For, if a fruitful exchange of information is to be possible between such ontologies and the data annotated in their terms, then each of the various systems involved must treat their relations in the same way. A relational expression must always stand for one and the same relation, even when it is used in multiple ontologies.

With regard to the most basic ontological relations (such as the subtypeor class-inclusion-relation and the part-whole relation), it is apparent in the literature that the same expressions are not always taken to stand for equivalent relations from one ontology to the next. In some places, this confusion goes so far that these basic relations are not distinguished from each other at all. For example, at one time, the medical terminology database Unified Medical Language System contained the statement plant leaf is_a plant and the SNOMED CT vocabulary contained the statement both uteri is_a uterus (see UMLS, see National Library of Medicine, 2006; SNOMED, 2007). ${ }^{50}$ The methodology that we are introducing will enable us to provide rigorous and unambiguous definitions for relational expressions between general terms, so that the meaning of these expressions can be stated precisely and used consistently.

\footnotetext{
${ }^{50} \mathrm{By}$ the time this chapter reaches publication, it may be that some, or all, of these statements have been rectified.
} 
In much of the literature on knowledge representation (see for example Fellbaum, 1998), relations between universals are presented as simple or basic, without any further specification. But this sort of treatment ignores essential aspects of the way in which instances in reality relate to one another. It is often not a trivial matter to determine whether or not a certain relation, in fact, obtains between the corresponding universals. For example, because there are female as well as male humans, we can certainly assert that human testicle part_of human, but not human has_ part human testicle. Because there are non-human mammals with hearts, human has_part heart is true, but not heart part_of human (a pig's heart, for example, is a part of a pig). For similar reasons, it is not true that growth has participant human. The temporal dimension can also be a source of special problems in determining whether relations apply. For example, even though every instance of the universal adult did at one time instantiate the universal child, it is not true that adult is_a child.

Unfortunately, many ontologies in the biological domain contain expressions for relations between universals whose correct usage can only be discerned through hints and loosely formulated suggestions. Without rigorous definitions, the logical connections between relations remain in the dark, and relational expressions become subject to multiple interpretations both within the same ontology (compare Ceusters, et al., 2004) and also from one ontology to the next (Smith, et al., 2004).

\section{Types of Relations}

Any relation to which our methodology can be applied (including the examples we discuss) must fulfill the following four criteria.

First, the relations in question must be genuine ontological relations. This means that they obtain between entities in reality, independently of our experience or methods of learning about them. They are also independent of the ways in which we represent them or make our knowledge about them processable by computers. This is by no means the case for all relations. For example, the Gene Ontology uses the relation $A$ annotates $B$. This relation is used to link certain genes or gene products with expressions from a controlled vocabulary. This relation does not link universals of biomedical reality as they are in themselves; rather, we use it to assert that a certain link has been effected by humans between a term in an ontology and (for example) some protein. Thus, it is not an ontological relation. 
Second, the relations of interest here are those domain-neutral relations which could appear, in principle at least, in any biomedical ontology. Certainly there are relations which hold between entities of specific types belonging to some specific domains. An example of this sort of specific relation would be $A$ is_genome_of $B$, which might be used in a genesequence ontology. Our strategy, however, is to define, as far as possible, a small set of high-level relations in a domain neutral way, and to construct definitions for low-level domain-specific relations on this basis.

Third, the relations must obtain universally. A statement of the form $A$ relation $B$ must obtain for all instances of $A$, and not just (for example) for some statistically representative selection. In many cases, the relations with which we have to deal will express analytic connections between universals, which is to say connections that can be understood to obtain universally when we analyze the corresponding general terms with which these universals are represented: for example, in cases such as skin cell is a cell or heart attack has_participant heart. Propositions expressing this kind of connection are true solely in virtue of the meanings of the terms involved and of the expressions which connect them. We do not have to examine the world in order to find out that, if something is a skin cell, then it is also a cell. While human beings do not need to be instructed on such matters, such instruction is needed by the automatic reasoning systems towards which ontologies are addressed.

Fourth, the relation must be definable in a simple, yet rigorous, way. This criterion is important since there are many relations that ontologists use for which they have, at best, only intuitive definitions or, sometimes, no definitions at all. Consider the relations physically_related_to and functionally_related_to, from the UMLS Semantic Network (National Library of Medicine, 2006). The former is defined as meaning: 'related by virtue of some physical attribute or characteristic', while the latter means 'related by the carrying out of some function or activity'. In neither case could we apply these definitions effectively in such a way that they would help in determining whether a given example is or is not an example of one or other of the relations in question. In neither case could we use the resultant assertions for computer-aided reasoning.

We insist upon this fourth criterion because we want to introduce definitions that are easy to understand, and which can be used effectively by humans. But, at the same time, we need definitions that can be used to support logic-based computer reasoning processes and help to support consistency from one ontology to the next. 
As we mentioned above, a biological ontology should represent only type-level relations of the form <universal, universals. The creation of rigorous definitions of these relations requires, however, first an understanding of those more basic relations which are of the forms $<$ instance, instance $>$ or $<$ instance, universal $>$. A $<$ universal, universal $>$ relation obtains only because a certain <instance, instance $>$ relation obtains between the universals' respective instances, or because a certain <instance, universal $>$ relation obtains between a universal and its instance. This sort of characterization of the relations between universals is anchored in the Aristotelian interpretation of the notion of universal, according to which universals exist only in their instances.

\section{Types of Relations and Limitations to the Use of Relational Expressions}

Before we can begin to define some basic relational expressions for a biological ontology, we must specify which expressions we will use to designate entities that stand as relata in corresponding relations. To do this, we must be able to speak of instances, as well as universals, in an appropriate way. We will avail ourselves of the tools of logic, including variables and quantifiers (see for example Hodges, 2001, and Chapter 5 above). Variables of various sorts are placeholders, respectively, for instances and universals of continuants, processes, and points in time:

$C, C_{1}, \ldots, C_{\mathrm{n}} \quad$ stand for continuant universals;

$P, P_{1}, \ldots, P_{\mathrm{n}} \quad$ stand for process universals;

$c, c_{1}, \ldots, c_{\mathrm{n}} \quad$ stand for instances of continuants;

$p, p_{1}, \ldots, p_{\mathrm{n}} \quad$ stand for instances of processes;

$r, r, \ldots, r_{\mathrm{n}} \quad$ stand for three-dimensional spatial regions;

$t, t_{1}, \ldots, t_{\mathrm{n}} \quad$ stand for points in time.

Continuants and processes form mutually exclusive categories. Continuants can be material entities such as a molecule, a cell, or a human being; but they can also be immaterial, such as a hole or a conduit. Immaterial continuants have some traits in common with spatial regions (Casati and Varzi, 1994) but can be distinguished from them in that they are immaterial parts of organisms. Just like material continuants, they move with the movement of their bearers from one spatial region to another. 
Biology occupies itself mainly with three-dimensional continuants, which typically have tops and bottoms, insides and outsides, fronts and backs. Processes, by contrast, have a beginning, middle, and an end. In contrast to continuants, processes unfold themselves along a temporal axis so that, for example, your childhood and your adulthood are temporal parts of the process that is your life.

Here, the concern is with two different, complementary perspectives on the same reality: one space- and matter-oriented, the other time- and change-oriented. There are certain logical and ontological relations between these perspectives, which we make explicit in our treatment of the relations by taking account of spatial regions and points in time (see Smith and Grenon, 2004). It follows from our approach, which recognizes a radical distinction between continuants and occurrents, that there are limitations on which sorts of entities can serve as relata in any given relation. For example, it is incoherent to form a relational statement of the form $P$ is_a $C$, because the subsumption relation cannot obtain between entities from incompatible categories. By contrast, the has participant relation (as in apoptosis has participant cell) requires that the first-named entity be a process universal and the second a continuant universal, rendering this relation something like a bridge between our two perspectives on reality.

Also, we need to distinguish between two types of relations at the level of instances: there are relations between continuants (for example: Mary's uterus is an instance level part of Mary), whose representation must contain a reference to points in time, and there are relations between processes, for whose representation this is not required. (The course of Mary's pregnancy is a part of Mary's life in a time-independent sense of parthood.) Since processes unfold through time, it is as if they already contain a reference or anchorage to the temporal dimension within themselves.

The placement of the relata in the ontological sextet, effectively, establishes the limitations as to which types of relations can obtain between which relata. An instantiation relation can only obtain between a particular and a universal, a participation relation only between a continuant and a process, and so forth. 


\section{Primitive Relations at the Level of Instances}

In order to be able to define the subsumption (is_a), part-whole, and participation relations at the level of universals, we must first list those relations which are not further definable and, therefore, which we view as primitive. Otherwise, we will be threatened either by an infinite regress (since each definition will require ever new, more basic vocabulary for its formulation) or a circular structure (where we effectively define an expression with the help of other expressions in which the expression to be defined already appears). The selected primitive relations should be evident, self-explanatory, and neutral with respect to the various domains of science. Hence, they are relations of the sort which obtain, not only within the field of the biology, but in any domain whatsoever. Except for the instantiation relation (instance_of), which obtains between an instance and a universal, all of the primitive relations obtain between instances; we can then use these primitive relations to define the relations at the level of universals.

We select the following relations as primitive, drawing in large part on the results outlined in (Smith, et al., 2005):

$c$ instance_of $C$ at $t$ : a primitive relation between a continuant-instance and a universal which it instantiates at a given point in time. This relation corresponds to the instantiation relation in the ontological sextet which obtains between a substance particular and a substance universal.

$p$ instance_of $P$ : a primitive relation between a process-instance and a universal which it instantiates independently of time. This relation corresponds to the instantiation relation in the ontological sextet which obtains between an individual process and a process universal.

$c$ part_of $c_{1}$ at $t$ : a primitive part-whole relation between two continuant instances and a time at which the one is part of the other.

$p$ part_of $p$ : a primitive part-whole relation which, independently of time, obtains between two process-instances (one is a processual part, or segment, of the other). 
$c$ located_in $r$ at $t$ : a primitive relation between a continuant instance, a 3 -dimensional spatial region which this instance occupies, and a time at which this instance occupies this region.

$p$ has_participant $c$ at $t$ : a primitive relation of participation among a process, a continuant, and a point in time. This is the inverse of the participation relation in the ontological sextet, which obtains at a certain point in time between a substance particular and an individual process.

For a human reader, these relations are relatively easy to understand. But in order to use them for computer applications, the meanings of the relational expressions must be rigorously characterized by means of axioms. Work on these axioms is not yet complete, but here are a few important ones. For the instance_of relation, the following axioms hold. This relation applies only to an instance and a universal, in that order. No entity can be simultaneously an instance and a universal. For the part_of relation, we have the following (Simons, 1987): This relation is irreflexive: no entity is a part of itself. It is anti-symmetric: if $x$ stands in the part_of relation to $y$, and $y$ stands in the part_of relation to $x$, then $x$ and $y$ are identical. It is transitive: if $x$ stands in the part_of relation to $y$, and $y$ in this relation to $z$, then $x$ stands in this relation to $z$. An additive principle holds, which guarantees the existence of mereological sums or wholes. A principle of differentiation holds: if $x$ stands to $y$ in the part_of relation and if $x$ and $y$ are not identical, then there exists a further part $z$ of $y$, which has no parts in common with $x$. The corresponding axioms for the instancelevel part-whole relation between continuants, must be modified in such a way that they contain a temporal index.

\section{Formal Definitions of Relations at the Level of Universals}

We now have at our disposal the instruments with which we can define the relational expressions that were our original goal: those between universals.

\subsection{The Definition of the Subsumption Relation}

The is $a$ relation is often identified with the relation of set inclusion, which is well-known from mathematical set theory. Under this mistaken interpretation, the instance_of relation corresponds to the set-theoretical 
relation of class membership. In this case a definition of $A$ is_a $B$ would be conceivable:

A is_a $\mathrm{B}={ }_{\text {def }}$ For all $x$ : if $x$ instance_of $A$, then $x$ instance_of $B$.

Unfortunately, this interpretation can deliver, at most, a necessary condition for the truth of $A$ is_a $B$. Two arguments speak against its acceptability as a sufficient condition.

(1) This interpretation permits cases in which the subsumption relation holds not between universals or types, but between merely contingent groupings of entities, as for example in: Cell nucleus in $10 \mathrm{ml}$ test tube is_a cell nucleus. This relation certainly holds without exceptions. But a cell nucleus in a $10 \mathrm{ml}$ test tube is not a special kind of cell nucleus, any more than a man wearing a hat, a man not swimming, or a man in Leipzig, is a special kind of man. Certainly reasoning systems of the sorts for which ontologies are useful will need to reason with such defined classes, and such defined classes will be indispensable for the handling of information about scientific or clinical investigations. But defined classes are to be distinguished, nonetheless, from the universals which form the subjectmatter of scientific theory.

(2) The temporal aspect receives no attention in this interpretation. One reason why 'cell nucleus in a $10 \mathrm{ml}$ test tube' does not designate a universal is that the predicate in question can both apply and fail to apply to the very same entity without the latter having changed in any way. This means that the interpretation may yield false results when applied to continuant universals, as in the already mentioned case: Adult is_a child.

We can take care of problem (1) by allowing only those type-level relations to express the sorts of genuine is_a relations which should be asserted in an ontology that correspond to statements of biological science.

Problem (2) can be dealt with by acknowledging the temporal aspect in is_a relations between continuant universals. Continuants, as opposed to processes, can instantiate various different universals while maintaining their identity over the course of their existence. Thus, we must distinguish two types of is_a relation: the is_a relation between continuants includes a temporal index, and the $i$ s_a relation between processes, which is timeindependent. We define:

$C$ is_a $C_{1}=$ def. for all $c$, $t$, if $c$ instance_of $C$ at $t$, then $c$ instance_of
$C_{1}$ at $t$. 
$P$ is_a $P_{1}=_{\text {def. }}$ for all $p$, if $p$ instance_of $P$, then $\mathrm{p}$ instance_of $P_{1}$.

\subsection{The Definition of the Part-Whole Relation}

Two kinds of part_of relation can also be distinguished at the level of universals, depending upon whether the relation obtains between continuants or processes. For continuants, $C$ part_of $C_{l}$ holds if and only if every instance of $C$ at each point in time stands in the part_of relation (at the level of instances) to some instance of $C_{l}$; for example: Cell nucleus part_of cell. We thus define:

$C$ part_of $C_{l}==_{\text {def. }}$ For all $c, t$, if $c$ instance_of $C$ at $t$, then there is a $c_{l}$, of which it holds that $c_{1}$ instance_of $C_{1}$ at $t$ and c part_of $c_{1}$ at $t$.

$C$ part_of $C_{1}$ says that instances of $C$, whenever they exist, exist as parts of instances of $C_{1}$. And analogously for processes: $P$ part_of $P_{1}$ holds if and only if on the level of instances every instance of $P$ stands in the part_of relation to at least one instance of $P_{1}$; for example: Childhood part_of life. $P$ part_of $P_{1}$ says that instances of $P$ always exist as parts of instances of $P_{1}$. We thus define:

$P$ part_of $P_{1}=_{\text {def. }}$ For all $p$, if $p$ instance_of $P$, then there is a $p_{1}$, such that $p_{l}$ instance_of $P_{l}$ and $\mathrm{p}$ part_of $p_{1}$ hold.

The definitions used here have a common logical structure. Each consists of a universally quantified conditional formula containing an existentially quantified formula as part ('for every $x \ldots$ there is some $y$...'). This logical form we call the all-some-structure. It captures certain logical relations in which statements about part-whole relations stand to each other. It implies that the $A$ and the $B$ for example in $A$ part_of $B$ are treated differently. So, it cannot be concluded from human uterus part_of human that human has human uterus as part. For while all instances of human uterus are at every time at which they exist instance-level parts of some instance of human, it is not the case that all instances of human have, at every time they exist, instances of human uterus as parts. 


\subsection{The Definition of the Participation Relation}

The primitive relation has_participant, at the level of instances, connects a continuant, a point in time, and a process in which the continuant is in some way involved. This relation obtains for example if a particular cell is at a particular time involved in a particular process of cell transportation. For the definition of the has_participant relation at the level of universals we proceed in a way analogous to the above. We thus define:

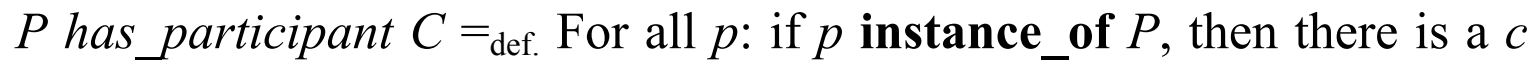
and a $t$, such that: $c_{1}$ instance_of $C_{1}$ and $p$ has_participant $c$ at $\mathrm{t}$.

Here, it should be noted that $P$ has_participant $C$ asserts merely that instances of $P$ require instances of $C$ as bearers. Because of the all-some structure of the definition of has participant, however, it does not follow that instances of $C$ are always involved in processes of a certain kind. It thus for example does not follow from human reproductive behavior has_participant human that all humans take part in human reproduction behavior.

\subsection{The Definition of the Location Relation}

The primitive relation $c$ located in $r$ at $t$ on the instance level holds between a continuant and its (unique) exact location at any given time. We can then derive a defined location relation between continuants, for example between a given cell nucleus and a given cell, as follows:

$c$ located_in $c_{1}$ at $t=$ def. for some $r, r_{l}, c$ located_in $r$ at $t$ and $c_{1}$ located_in $r_{l}$ at $t$ and $r$ part_of $r_{1}$.

In the relation $r$ part_of $r_{l}, r$ and $r_{l}$ can be conceived of as special cases of instances of continuants. This relation comprises both the relation of exact location between two continuants if $r$ and $r_{l}$ are identical and the relation of inexact location between two continuants if $r$ is a proper part (for details on the proper-part relation, see Chapter 8) of $r_{1}$. An example of the former relation is that between a gas and a cavity that it fills completely; an example of the latter is the location relation between a testicle and a scrotum. In this manner we arrive at a formal definition for the location relation at the level of universals: 
$C$ located_in $C_{1}=$ def. for all $c$, $t$, if $C$ instance_of $c$ at $t$ then there is some $c_{1}$ such that: $C_{1}$ instance_of $c_{1}$ at $t$ and $c$ located_in $c_{1}$ at $t$.

\section{The Logic of Relations}

The inverse relation $R^{-1}$ of a two-place relation $R$ is defined as the relation that obtains between a pair of relata if and only if the original relation $R$ obtains when the order of relata is reversed. It is easy to define inverse relations of the instance-level primitive relations which we have discussed. The definition of the inverse relation to is_a, obtaining between a universal and a universal, is trivial as well:

A has_subclass $\mathrm{B}=_{\text {def. }} \mathrm{B}$ is_a $\mathrm{A}$.

Adding the has_subclass relation does not benefit us in increasing the expressive power of an ontology: each piece of information that can be expressed with the help of the has_subclass relation can be expressed with the is $a$ relation. When we move to the other relations at the level of universals, whose definitions have an all-some structure, then this is not the case. Consider, for example, the relations of parthood. At the instance level $x$ part_of $y$ is true if and only if its inverse, $y$ has_part $x$, is true. At the level of universals, however, this is not the case. Thus, for example, although the relational statement human testicle part_of human is true, since every instance of human testicle is part of some instance of the universal human, there is no corresponding relation with an all-some structure which links every instance of human with at least one instance of human testicle. This should not be seen as a deficit in our definition of the relations, but rather as a reflection of the way in which reality is constructed.

Nonetheless, a has part relation at the level of universals that is defined in the all-some way is useful and important, for such a relation can be used to express propositions such as human has part heart. The type-level has part relation we need is not, however, an inverse of the part_of relation, as is seen in the fact that we have:

cell has_part nucleus

but not:

nucleus part_of cell. 
Corresponding to our two part_of relations above, we have two has_part relations as follows:

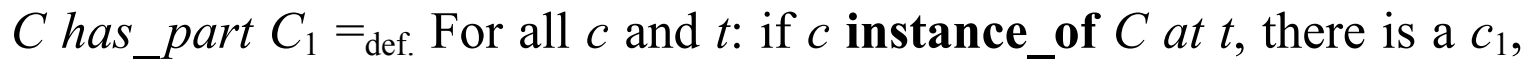
such that $c_{1}$ instance_of $C_{1}$ at $t$ and $c_{1}$ part_of $c$ at $t$.

$P$ has_part $P_{1}==_{\text {def. }}$ For all $p$ : if $p$ instance_of $P$, there is a $p_{1}$ such that $p_{1}$ instance_of $P_{1}$ and $p_{1}$ part_of $p$.

In contrast to the has_subclass relation referred to above, the has_part relations thus defined bring about an increase in expressive power. They allow the representation of relations at the type level which could not be captured using part_of alone.

The characteristics of the relations at the level of universals with respect to transitivity, reflexivity, and symmetry are presented in the following table.

Figure 1: Logical attributes of some formal relations.

\begin{tabular}{|c|c|c|c|c|}
\hline Relation & Transitive & Symmetrical & Reflexive & Antisymmetric \\
\hline is_a & + & - & + & + \\
part_of & + & - & + & + \\
\hline has_participant & - & - & - & - \\
\hline
\end{tabular}

\section{Conclusion}

Simple and rigorous definitions of relational expressions make it possible to render their meaning in a lucid way. For example, it is obvious which relation an expression such as 'is_a' stands for. Further, the form of the definition enables us clearly to see, not only the limitations on the potential relata of the relation, but also the logical properties of the relations between universals within an ontology. Our definitions are formulated in such a way that they enable a unified treatment of the corresponding 
relational expressions in all biological ontologies. The methodology used to create these definitions can be used in all facets of biological science, as well as other scientific domains. In this way, it can make a contribution to the project of rendering the multiplicity of biological ontologies interoperable, even though many of these ontologies were developed for entirely different reasons and with entirely different goals. Our methodology allows for the application of automated inference mechanisms as well. Nevertheless, there is still much work to be done in axiomatizing primitive relations. 